\title{
¿ES POSIBLE MEJORAR EL AUTOCONCEPTO FÍSICO UNIVERSITARIO MEDIANTE UNA INTERVENCIÓN COGNITIVA?
}

\author{
IS IT POSSIBLE TO ENHANCE THE PHYSICAL \\ SELF-CONCEPT OF UNIVERSITY STUDENTS' \\ USING A COGNITIVE INTERVENTION?
}

\author{
INGE AXPE ${ }^{1}$, GUILLERMO INFANTE BORINAGA ${ }^{1} \mathrm{Y}$ \\ ARANTZA FERNÁNDEZ ZABALA ${ }^{1}$
}

Cómo referenciar este artículo/How to reference this article:

Axpe, I., Infante Borinaga, G. y Fernández Zabala, A. (2015). ¿Es posible mejorar el autoconcepto físico universitario mediante una intervención cognitiva? [Is it possible to enhance te physical self-concept of university students' using a cognitive interventions?]. Acción Psicológica, 12(1), 23-34. doi: http://dx.doi.org/10.5944/ap.12.1.11864

\section{Resumen}

El interés de las últimas décadas por conocer los factores implicados en el bienestar de los sujetos ha mostrado la estrecha relación entre la satisfacción vital y el autoconcepto físico. Contribuir a mejorar la autopercepción física de las personas, especialmente adolescentes y jóvenes, mejoraría tanto su salud física como mental, repercutiendo positivamente sobre su satisfacción vital. Pese a ello, no se conocen propuestas de intervención que desde una perspectiva multidimensional y cognitiva, traten de mejorar el autoconcepto físico de los jóvenes. En este estudio se evalúa la aplicación de un programa de estas características con 171 estudiantes de universidad; 112 sujetos en el grupo experimental (edad media 20.00, DT $=3.36)$ y 59 en el grupo control (edad media 20.10, DT $=3.44$ ). Los re- sultados no indican mejoras estadísticamente significativas de los sujetos tras la intervención, no obstante ofrecen una información relevante que contribuye a perfilar las características a incluir en el diseño de futuras implementaciones más eficaces y exitosas.

Palabras clave: autoconcepto físico, intervención cognitiva; programa psico-educativo; estudiantes universitarios.

\section{Abstract}

The interest raised during the last decade about the factors involved in the personal well-being has shown the positive relation between life satisfaction and physical self-concept. Improving the physical self-perception of people, especially during adoles-

\footnotetext{
Agradecimientos: Investigación desarrollada dentro de la actividad del Grupo Consolidado de Investigación IT701-13 del Gobierno Vasco, y el proyecto de investigación EDU2009-10102 (subprograma EDUC) “Autoconcepto (multidimensionalidad y estructura jerárquica) y ajuste psicosocial.
}

Correspondencia: Inge Axpe. Universidad del País Vasco. Email: inge.axpe@ehu.eus

${ }^{1}$ Universidad del País Vasco

Recibido: 13 de febrero de 2014

Aceptado: 25 de abril de 2015 
cence and youth, would contribute both to their physical and mental health, and therefore, boost their life satisfaction. Despite, there is a lack of intervention proposals which, from a multidimensional and cognitive approach, try to improve the physical self-concept of young people. This study presents and evaluates a program of those features, implemented with 171 university students (112 in the experimental group, mean age 20.00 years, SD $=3.36$; and 59 students in the control group, mean age 20.10 years; $S D=3.44)$. After the completion of the program, no statistically significant differences are found in the physical self-concept scores of the experimental group. Nonetheless, those results suppose relevant information in order to identify the more effective characteristics which future intervention designs should consider to booster their efficacy.

Keywords: Physical self-concept; cognitive intervention; psycho-educative program; university students.

\section{Introducción}

En las últimas décadas, desde la perspectiva de la psicología positiva, la investigación ha mostrado su interés por el estudio de aquellos factores que promueven el bienestar (Góngora, 2010), tratando así de promover el ajuste y funcionamiento óptimo de los sujetos a través de la identificación de sus fortalezas (GonzálezBrignardello y Sánchez-Elvira-Paniagua, 2013). El autoconcepto ha cobrado un papel esencial en dicho propósito, ya que las autopercepciones y actitudes que las personas guardan de sí mismas y de su entorno han mostrado una estrecha relación con el nivel de satisfacción vital de éstas (Videra-García y Reigal-Garrido, 2013); satisfacción y bienestar que contribuye y se relaciona con la felicidad y la salud mental (GarcíaAlandete y Bernabé-Valero, 2013). Efectivamente, el que jóvenes y adolescentes no logren configurar una adecuada percepción de su yo (Sánchez-Quintero y De la Vega, 2013) se asocia a diversos trastornos y, por supuesto, a un considerable malestar psicológico y personal.

El autoconcepto es una representación mental compleja, multidimensional y jerárquicamente organizada que las personas construyen de su yo. Según la propuesta originaria de Shavelson, Hubner y Stanton (1976), dicha representación, surgida de la percepción individual formada a partir de la experiencia con el entorno, las interacciones con otros significativos, y las atribuciones del propio comportamiento, estaría constituida por diversos dominios que a su vez se compondrían de distintas dimensiones. El autoconcepto físico sería precisamente uno de los dominios que contribuiría en mayor medida a conformar el autoconcepto general (Fernández, Contreras, García, y González, 2010; Goñi, y Infante, 2010), y que cómo éste, ha mostrado una estrecha relación con el bienestar y satisfacción con la vida (Borrego, LópezSánchez y Díaz-Suárez, 2012; Haugen, Ommundsen y Seiler, 2013). Entre los distintos modelos explicativos propuestos en relación al autoconcepto físico, cabe destacar, por su parsimonia y poder explicativo, aquellos que postulan cuatro dimensiones constitutivas: la habilidad física, el atractivo físico, la fuerza y la competencia deportiva (Fox y Corbin, 1989) o la condición física (Goñi, Ruiz de Azúa, y Rodríguez, 2006; Esnaola y Goñi, 2006). De entre ellas, la dimensión de atractivo físico ha demostrado ser una de las que más incide sobre el autoconcepto general, especialmente en el caso de las mujeres (Fernández-Bustos, González-Martí, Contreras, y Cuevas, 2015; Lindwall, Asci, Crocker, y Teixeira, 2014), observándose también su peso en el bienestar subjetivo, pues constituye un importante factor de riesgo de depresión adolescente (Franko y Striegel-Moore, 2002).

Son varios los factores a tener en cuenta a la hora de explicar esta estrecha relación. Por un lado, la fuerte presión social respecto a la imagen y la belleza presente en los medios de comunicación (Bermejo, Saúl y Jenaro, 2011), en la que se exhiben cuerpos inalcanzables y extremamente delgados en mujeres o musculados en hombres (Esnaola, Rodríguez y Goñi, 2010), parece la responsable de que el atractivo físico se revele como una de las dimensiones del autoconcepto físico que más parece determinar la satisfacción vital (Contreras, Fernández, García, Palou y Ponseti, 2010; Goñi e Infante, 2010; Infante, Goñi y Villarroel, 2011; Parker, Martin y Marsh; 2008). La incapacidad por alcanzar o aproximarse a estos modelos de belleza puede provocar una fuerte insatisfacción con el propio cuerpo y el propio yo, empujando a los sujetos a la realización de conductas nocivas o de riesgo (Esnaola et al., 2010) y/o al desarrollo de trastornos de la conducta alimentaria (Bermejo et al., 2011), cuya relación con un bajo autoconcepto ha sido ya sobradamente probada (Esnaola, 2007; Goñi y Rodríguez, 2007).

Por otro lado, es bien conocida la relación directa y positiva entre autoconcepto físico y una práctica de la actividad física moderada y automotivada (Contreras et al., 2010; Fernández et al., 2010; Infante, Axpe, Revuelta y Ros, 2012) por lo que es probable que el mantenimiento de hábitos y conductas saludables como la práctica de actividad física incidan de manera indirecta sobre la satis- 
facción con la vida a través y gracias al autoconcepto físico (Goñi e Infante, 2010). De hecho, tienden a ser las personas con mejor autoconcepto físico aquellas que muestran mayor implicación y adherencia a las actividades deportivas (Annesi, Unruh, Marti, Gorjala y Tennant, 2011; Borrego et al., 2012; Fernández et al., 2010), por lo que el autoconcepto físico se torna esencial de cara a potenciar y aumentar la realización de dichas actividades (Scarpa, 2011; Vancampfort et al., 2011; Vedul-Kjelsås, Sigmundsson, Stensdotter y Haga, 2012), tan necesarias para evitar enfermedades crónicas, hábitos nocivos de inactividad o consumo de sustancias (Ceschini, Andrade, Oliveira, Araujo y Matsudo, 2009), así como para promocionar la salud mental (Haugen et al., 2013) y el bienestar (Guedes, Souza, Ferreirinha y Silva, 2012; Rahmani-Nia, Damitchi, Azizi y Hoseini, 2011).

La adolescencia y temprana juventud constituyen periodos de cambio y transición fundamentales para los sujetos, de tal manera que cobra especial relevancia el atender su salud, tanto física como psicológica (VideraGarcía y Reigal-Garrido, 2013), pues podría condicionar el bienestar futuro de estas personas. Mejorar y trabajar a favor de su autoconcepto físico resulta, por tanto, primordial, y son diversos los estudios que muestran estos esfuerzos a través del diseño e implementación de programas que buscan implicar a los sujetos participantes en la realización de diversos tipos de entrenamiento y actividades físicas (Annesi et al., 2011; Haugen et al., 2013; Lubans, Aguiar y Callister, 2010; Schmidt, Valkanover, Roebers y Conzelmann, 2013; Velez, Golem y Arent, 2010).

No obstante, la práctica deportiva puede resultar contraproducente cuando ésta se realiza con el objetivo de mejorar la propia imagen (Fernández et al., 2010), estando en estos casos vinculada a un peor autoconcepto físico. Algo que además coincide con el hecho de que el atractivo físico parece ser, en algunos estudios, la autopercepción más resistente a la mejora o cambio a través de la realización de actividad física (Contreras et al., 2010).

Las mujeres son los sujetos que más relevancia parecen conferir a la dimensión del atractivo físico (Molero, Zagalaz-Sánchez y Cachón-Zagalaz, 2013; Soriano, Navas y Holgado, 2011), lo que explicaría la mayor incidencia de Trastornos de la Conducta Alimentaria en esta población (Jenaro, Flores, Bermejo y Cruz, 2011), así como su tendencia a mostrar puntuaciones inferiores a las de los hombres en autoconcepto físico (MayorgaVega, Viciana y Cocca, 2012; Soriano, Sampascual y Navas, 2010).
Precisamente, intervenciones desarrolladas con estudiantes universitarias para la prevención de estos trastornos (Anuel, Bracho, Brito, Rondón y Sulbarán, 2012; Gasco, Briñol y Horcajo, 2010; Stice, Marti, Rohde y Shaw, 2011; Stice, Rohde, Durant y Shaw, 2012) han mostrado la posibilidad de reducir la insatisfacción corporal mediante propuestas en las que, lejos de promocionar la actividad física, se potencia, entre otros, la revisión crítica de los medios de comunicación o se recurre a la teoría de la disonancia cognitiva (ver Góngora, 2010). Se pretende, por medio de estos programas interactivos (Stice y Shaw, 2004) que incorporan actividades de discusión, descubrimiento guiado y técnicas cognitivas; provocar una revisión, reflexión crítica y análisis (Escoto, Camacho, Rodríguez y Mejía, 2010) que logre implicar y así provocar un cambio en la forma de pensar e interpretar la información de los sujetos. Este tipo de programas se tornan herramientas fundamentales en los esfuerzos por promocionar el autoconcepto físico de las personas, al resultar de fácil implementación y bajo coste (Celio et al., 2008), siendo por tanto adecuados para su uso en grupos grandes y distintos tipos de poblaciones.

Es bien cierto que no todos los programas implementados desde una perspectiva cognitiva obtienen los beneficios esperados (Nicolino, Martz y Curtin, 2001; O'Brien y LeBow, 2007), sin embargo, el hecho de que sea factible su implementación y exista la posibilidad de mejorar aspectos concretos de las autopercepciones de estudiantes universitarios, lleva a la necesidad de avanzar un paso más y abordar el autoconcepto físico en su totalidad, incorporando una perspectiva multidimensional, básica para asegurar la eficacia de la intervención (Cava, Musitu y Vera 2000) al atender todas y cada una de las dimensiones que constituyen dicho constructo. Se ha comprobado que con alumnado universitario (Axpe, Goñi e Infante, 2013) una intervención cognitiva multidimensional puede resultar enormemente eficaz si se acompaña, en consonancia a las directrices de diversos autores (Marsh y Craven, 2006; Marsh, Papaioannou y Theodorakis, 2006), de una práctica deportiva intensa y frecuente.

Resulta imprescindible, no obstante, comprobar si una intervención de más fácil implementación y diseminación, carente de entrenamiento físico y centrada por tanto en el trabajo cognitivo, podría resultar igualmente eficaz. Aplicaciones previas del programa (Axpe et al., 2013) con alumnado universitario de grado de Educación Física mostraron un significativo aumento de la autopercepción física de los sujetos participantes al finalizar el programa. Sin embargo, sería preciso contar con datos de una muestra más representativa de la población general, exenta de formación y práctica deportiva dentro de su formación universitaria, para comprobar el efecto de participar en 
una intervención cognitiva para la mejora del autoconcepto físico. Intervenciones de estas características supondrían un enorme beneficio social con un muy bajo coste, contribuyendo a promocionar la autopercepción física de jóvenes y adolescentes, especialmente mujeres, más afectadas por su imagen y atractivo físico y quizá por ello, menos tendentes a la implicación en actividades físico deportivas, pues el autoconcepto físico es también un importante determinante de la realización de actividad física (Babic et al., 2014).

Así pues, en el presente estudio se muestra la implementación de un programa psico-educativo, el "Mírate bien" (http://www.psikor.es/index.php) desde una perspectiva exclusivamente cognitiva y atendiendo la multidimensionalidad del autoconcepto físico, ya que a través de sus seis etapas trabaja sus cuatro dimensiones constitutivas: habilidad, condición, atractivo físico y fuerza (Esnaola y Goñi, 2006; Fox y Corbin, 1989; Goñi, Ruiz de Azúa, y Rodríguez, 2006) ocupándose también de aquellos factores que la investigación previa ha mostrado estrechamente vinculados a las mismas: los hábitos saludables de vida (Revuelta et al., 2013), los influjos socioculturales (Rodríguez-Fernández, González-Fernández, y Goñi, 2013) y la propia interpretación subjetiva utilizando estrategias diversas como el establecimiento de marcos de referencia adecuados (Chanal, Marsh, Sarrazin, y Bois, 2005). Mediante la implementación del programa se pretende establecer su eficacia en la mejora del autoconcepto físico centrándose en aspectos cognitivos (información, toma de conciencia, identificación de pensamientos, reestructuración cognitiva, motivación para el cambio...) y sin implementar un entrenamiento físico adicional, para comprobar el efecto de un tipo de intervención de dichas características sobre el autoconcepto físico de alumnado universitario, estudiando también su posible impacto diferencial en función de sexos. Se espera que la implementación del programa:

a) Tenga un impacto positivo en la mejora de las puntuaciones de autoconcepto físico de los sujetos.

b) Suponga un mayor beneficio para las mujeres que para los hombres, al tener éstas un menor autoconcepto físico por la mayor presión social recibida.

\section{Método}

\section{Participantes}

En este estudio de diseño cuasi-experimental pretest-postest con grupo control, tomaron parte 171 estu- diantes (45 hombres y 126 mujeres, el $26.3 \%$ y $73.7 \%$ de la muestra, respectivamente) de la Universidad del País Vasco/Euskal Herriko Unibertistatea (UPV/EHU), cursando las titulaciones de Educación Primaria y Educación Social. Entre éstos, 112 estudiantes participaron como sujetos experimentales (media de edad de 20.00, $\mathrm{DT}=3.36)$, mientras que 59 sujetos formaron el grupo control (media de edad 20.10, DT $=3.44$ ).

\section{Procedimiento}

El alumnado fue informado a través de la docente de la asignatura de Psicología de la Educación de la posibilidad de participar en el estudio. Se solicitó además su consentimiento para la cumplimentación y recogida de información relativa al autoconcepto físico de manera previa y posterior a la intervención. Aquellas personas interesadas recibieron los materiales constituyentes del programa y se estableció con ellas un plan de trabajo en el que se combinó el trabajo autónomo e individual (seis etapas o entregas) con la interacción grupal (tres sesiones de dos horas de duración).

Para el trabajo individual se estableció un calendario de entrega quincenal, de manera que el alumnado, cada dos semanas, cumplimentó de manera autónoma las tareas y ejercicios propuestos en cada una de las seis etapas o unidades que constituyen el programa. Además, al finalizar cada etapa, cada estudiante realizó una redacción personal reflexionando en torno a los aspectos trabajados. La periodicidad de trabajo y entrega quincenal era acompañada de una revisión del trabajo autónomo, en la que se incluía feedback individual (por escrito) en relación al proceso.

En las sesiones grupales, cuyo objetivo era reforzar la implementación y trabajo autónomo, se abordaron las siguientes cuestiones: 1) la relevancia del autoconcepto físico en el bienestar personal y el mantenimiento de hábitos físicos saludables; 2) la influencia del entorno en la autopercepción física, prestando especial atención a los medios de comunicación; y 3) los mecanismos cognitivos adecuados e inadecuados en la percepción del yo físico. Las sesiones grupales se desarrollaron en cuatro grupos (dos para los estudiantes de Educación Social y dos para el alumnado de Educación Primaria) de entre 25-30 alumnos, conducidas por la misma persona, siguiendo un mismo diseño y realizando iguales actividades.

El grupo control no recibió ningún tipo de intervención, evaluándose el autoconcepto físico de sus sujetos en las mismas dos ocasiones en que fueron recogidas las 
puntuaciones de los participantes en el grupo experimental. Se aseguró el tratamiento confidencial de los datos a lo largo de todo el estudio.

\section{Instrumentos de medida}

Al tratarse de alumnado cursando sus estudios en lengua vasca, para la evaluación del autoconcepto físico se escogió el único instrumento diseñado y validado en tal lengua, el Autokontzeptu Fisikoaren Itaunketa (AFI) (Esnaola y Goñi, 2006; Esnaola y Zulaika, 2009). Este cuestionario postula cuatro dimensiones constituyentes del autoconcepto físico: habilidad física (ej., "No soy hábil en los deportes"), condición física (ej., "Tengo buena resistencia para la activad física"), atractivo físico (ej., "No estoy a gusto con mi apariencia física") y fuerza física (ej., "Soy fuerte"). Ofrece también dos escalas de orden superior: el autoconcepto físico general (ej., "Estoy satisfecho con mi físico") y el autoconcepto general (ej., "Estoy satisfecho conmigo mismo"), puntuándose sus ítems en una escala tipo Likert de 5 puntos $(1=$ Totalmente en desacuerdo, $5=$ Totalmente de acuerdo).

El cuestionario utilizado en este estudio muestra una adecuada fiabilidad de todas sus escalas (coeficiente alfa de Cronbach) con índices de .73 en el autoconcepto físico general y .79 en el autoconcepto general, .77 en las escalas de habilidad y de condición física, .76 en la escala de atractivo físico y .83 en la escala de fuerza. Sus cuatro factores explican $66 \%$ de la varianza y se ha comprobado su ajuste al modelo tetradimensional en muestras de ambos sexos y diversas edades (Esnaola y Goñi, 2006; Esnaola y Zulaika, 2009; Esnaola, Infante y Zulaika, 2011).

\section{Análisis de datos}

El análisis de los datos obtenidos se realizó mediante el paquete estadístico SPSS versión 19.0 estableciendo, para la interpretación de los resultados, el nivel de significación estadística en .05 .

Cabe señalar que la muestra a la que se refiere este estudio estaba constituida únicamente por aquellos cuestionarios cumplimentados de manera correcta (en su totalidad), prescindiendo de aquellos que mostraban ítems sin respuesta, por lo que no fue preciso realizar imputación alguna de los valores perdidos.
Para comprobar la eficacia de la implementación realizada, en primer lugar se contrastaron los supuestos de normalidad, mediante la prueba de KolmogorovSmirnov, y de homogeneidad de varianza a través de la prueba de Levene, para proceder posteriormente a la estimación de posibles diferencias significativas en las puntuaciones medias relativas al autoconcepto físico antes y después del tratamiento. En este caso, se recurrió a un análisis bivariante o de diferencias de medias (prueba $t$ de Student) para muestras no relacionadas en las comparaciones intergrupo (grupo experimental y control) y para muestras relacionadas en los contrastes intragrupo.

Debido a que en la comparación de puntuaciones en función de sexos los hombres mostraron un mejor autoconcepto que las mujeres antes ya de la intervención, se recurrió a un Análisis de Covarianza (ANCOVA) a fin de controlar dichas diferencias previas (Dimitrov y Rumrill, 2003).

Por último, se calculó el tamaño de efecto de la intervención, cuantificando de este modo la diferencia real entre grupos atribuible al programa (Sánchez-Meca, 2008) y evitando, por tanto, asumir erróneamente que las significatividad estadística representa el tamaño o magnitud de la eficacia del tratamiento seguido. Se obtuvo el índice de cambio medio tipificado dMR (standardized mean change index) cuando se estimó el tamaño del efecto dentro de cada grupo, y la diferencia media estandarizada, $d$ (standardized mean difference) para comprobar el tamaño del efecto de la intervención. En la interpretación de los tamaños de efecto se tomaron como referencia los valores $d=0.2$ como magnitud de efecto baja; $d=0.5$ magnitud media y $d=0.8$ magnitud alta (Cohen, 1988).

\section{Resultados}

Las puntuaciones medias de los sujetos del grupo experimental y control, ofrecidas en la tabla 1, al ser comparadas de manera previa a la implementación del programa, no reflejaron diferencias estadísticamente significativas: habilidad $(t=0.04, p=.97)$; condición $(t=-0.23$, $p=.82)$; atractivo $(t=0.37, p=.71)$; fuerza $(t=0.25, p=$ $.80)$; autoconcepto físico general $(t=-0.76, p=0.45)$ y autoconcepto general $(t=-0.80, p=0.43)$. 
Tabla 1

Estadísticos descriptivos y comparación de grupos en autoconcepto físico

\begin{tabular}{|c|c|c|c|c|c|c|c|c|c|c|c|c|c|c|}
\hline \multirow{4}{*}{ Escala } & \multirow{3}{*}{\multicolumn{2}{|c|}{$\begin{array}{r}\text { Pre- } \\
\text { tervención }\end{array}$}} & \multicolumn{7}{|c|}{ Comparación Pre-Post Intragrupo } & \multirow{2}{*}{\multicolumn{5}{|c|}{$\begin{array}{r}\text { Comparación } \\
\text { Post-intervención entre grupos }\end{array}$}} \\
\hline & & & \multirow{2}{*}{\multicolumn{2}{|c|}{$\begin{array}{r}\text { Post- } \\
\text { intervención }\end{array}$}} & \multirow{2}{*}{\multicolumn{5}{|c|}{ Tamaño efecto }} & & & & & \\
\hline & & & & & & & & & & \multicolumn{5}{|c|}{ Tamaño efecto } \\
\hline & M & $D T$ & M & $D T$ & $t$ & $p$ & $d_{\mathrm{MR}}$ & & C 95\% & $t$ & $p$ & $d$ & & C 95\% \\
\hline \multicolumn{15}{|l|}{ Habilidad } \\
\hline Experimental & 2.96 & 1.03 & 3.00 & 0.93 & -0.52 & 0.61 & 0.03 & -0.27 & 0.34 & -0.49 & 0.62 & 0.07 & 0.38 & -0.25 \\
\hline Control & 2.96 & 0.97 & 3.08 & 1.04 & -1.12 & 0.27 & 0.01 & -0.23 & 0.48 & & & & & \\
\hline \multicolumn{15}{|l|}{ Condición } \\
\hline Experimental & 3.68 & 0.99 & 3.70 & 0.95 & -0.34 & 0.74 & 0.02 & -0.28 & 0.32 & 0.20 & 0.84 & 0.07 & -0.25 & 0.38 \\
\hline Control & 3.72 & 1.07 & 3.67 & 1.04 & 0.41 & 0.69 & 0.05 & 0.37 & -0.27 & & & & & \\
\hline \multicolumn{15}{|l|}{ Atractivo } \\
\hline Experimental & 3.30 & 1.05 & 3.33 & 1.03 & -0.41 & 0.68 & 0.03 & -0.28 & 0.33 & 0.15 & 0.88 & 0.04 & 0.35 & -0.28 \\
\hline Control & 3.24 & 1.02 & 3.31 & 1.09 & -.55 & 0.59 & 0.07 & -0.26 & 0.39 & & & & & \\
\hline \multicolumn{15}{|l|}{ Fuerza } \\
\hline Experimental & 3.70 & 1.08 & 3.66 & 1.05 & 0.64 & 0.53 & 0.04 & 0.35 & -0.27 & -0.05 & 0.96 & 0.05 & 0.37 & -0.26 \\
\hline Control & 3.65 & 1.11 & 3.67 & 1.05 & -0.16 & 0.88 & 0.02 & -0.29 & 0.33 & & & & & \\
\hline \multicolumn{15}{|l|}{$\begin{array}{l}\text { Autoconcepto Físico } \\
\text { General }\end{array}$} \\
\hline Experimental & 3.70 & 0.96 & 3.76 & 0.86 & -1.05 & 0.29 & 0.06 & -0.27 & 0.39 & -0.44 & 0.66 & 0.06 & -0.26 & 0.38 \\
\hline Control & 3.82 & 1.03 & 3.82 & 0.97 & 0.00 & 1.00 & 0.00 & 0.31 & -0.31 & & & & & \\
\hline \multicolumn{15}{|l|}{$\begin{array}{l}\text { Autoconcepto } \\
\text { General }\end{array}$} \\
\hline Experimental & 4.17 & 0.91 & 4.14 & 0.91 & 0.40 & 0.69 & 0.03 & 0.34 & -0.27 & -0.54 & 0.59 & 0.03 & -0.28 & 0.35 \\
\hline Control & 4.28 & 0.84 & 4.22 & 0.83 & 0.54 & 0.59 & 0.07 & 0.39 & -0.26 & & & & & \\
\hline
\end{tabular}

Finalizada la intervención, el contraste entre las puntuaciones finales del grupo experimental con aquellas de los sujetos control, no reflejó cambios estadísticamente significativos en ninguna de las escalas: habilidad $(t=$ $0.49, p=.62)$; condición $(t=0.20, p=.84)$; atractivo $(t$ $=0.15, p=.88)$; fuerza $(t=-0.05, p=.96)$; autoconcep-

El contraste intragrupo en el grupo control tampoco reveló cambios estadísticamente significativos en ninguna escala: habilidad $(t=-1.12, p=.27)$; condición $(t=$ $0.41, p=.69)$; atractivo $(t=-0.55, p=.59)$; fuerza $(t=-$ $0.16, p=.88)$; autoconcepto físico general $(t=0.00, p=$ $1.00)$ y autoconcepto general $(t=0.54, p=.59)$. El cálculo del tamaño de efecto, nuevamente en todos los casos de muy baja magnitud (dMR entre 0.00 y 0.07 ), corroboró la ausencia de significación estadística atendiendo a los intervalos de confianza.

Para finalizar, en relación al sexo, se comprobó que efectivamente, los hombres mostraron puntuaciones más elevadas que las mujeres en todas las escalas del autoconcepto físico antes del tratamiento (ver tabla 2), siendo estas diferencias estadísticamente significativas en to físico general $(\mathrm{t}=-0.44, p=.66) \mathrm{y}$ autoconcepto general $(t=-0.54, p=.59)$. Resultados que fueron corroborados al calcular el tamaño de efecto correspondiente a cada escala, en todos los casos de muy baja magnitud ( $d$ entre 0.03 y 0.07 ) y con intervalos de confianza que reafirmaron la ausencia de significatividad estadística.

las dimensiones de atractivo $(t=2.00, p=.04)$; autoconcepto físico general $(t=2.44, p=.02) \mathrm{y}$ autoconcepto general $(t=2.04, p=.04)$. En vista de estos resultados, se procedió analizar un posible efecto diferencial del tratamiento en función de sexos, controlando para ello las puntuaciones inicialmente más elevadas de los hombres mediante un análisis de varianza univariante (ANCOVA). Los resultados, en ningún caso estadísticamente significativos, mostraron que no existe tal diferencia de eficacia: habilidad $(\mathrm{F}=0.03, p=.86)$; condición $(\mathrm{F}=1.95, p=$ $.17)$; atractivo $(\mathrm{F}=0.03 ; p=.86)$; fuerza $(\mathrm{F}=0.00, p=$ $.96)$; autoconcepto físico general $(\mathrm{F}=1.76, p=.19) \mathrm{y}$ autoconcepto general $(\mathrm{F}=3.14, p=.08)$. El cálculo del tamaño de efecto refrendó estos resultados, reflejando tanto para hombres como para mujeres, índices de muy baja magnitud y sin significatividad estadística. 
Tabla 2

Estadísticos descriptivos y comparación del autoconcepto físico en función de sexo

\begin{tabular}{|c|c|c|c|c|c|c|c|c|c|c|c|}
\hline \multirow{2}{*}{ Escala } & \multicolumn{4}{|c|}{ Pre-intervención } & \multicolumn{7}{|c|}{ Post-intervención } \\
\hline & M & $D T$ & $t$ & $p$ & $M$ & $D T$ & $d_{\mathrm{MR}}$ & IC & $5 \%$ & $F$ & $p$ \\
\hline \multicolumn{12}{|l|}{ Habilidad } \\
\hline Hombres & 3.23 & 1.00 & \multirow{2}{*}{1.51} & \multirow{2}{*}{0.13} & 3.19 & 0.85 & 0.04 & 0.34 & -0.27 & \multirow{2}{*}{0.03} & \multirow{2}{*}{0.86} \\
\hline Mujeres & 2.88 & 1.03 & & & 2.94 & 0.94 & 0.06 & -0.26 & 0.37 & & \\
\hline \multicolumn{12}{|l|}{ Condición } \\
\hline Hombres & 4.00 & 1.11 & \multirow{2}{*}{1.89} & \multirow{2}{*}{0.06} & 4.08 & 1.05 & 0.07 & -0.23 & 0.37 & \multirow{2}{*}{1.95} & \multirow{2}{*}{0.17} \\
\hline Mujeres & 3.59 & 0.93 & & & 3.59 & 0.89 & 0.00 & 0.29 & -0.29 & & \\
\hline \multicolumn{12}{|l|}{ Atractivo } \\
\hline Hombres & 3.65 & 1.24 & \multirow{2}{*}{2.00} & \multirow{2}{*}{$0.04^{*}$} & 3.60 & 1.20 & 0.04 & 0.35 & -0.27 & \multirow{2}{*}{0.03} & \multirow{2}{*}{0.86} \\
\hline Mujeres & 3.19 & 0.96 & & & 3.25 & 0.97 & 0.06 & -0.26 & 0.38 & & \\
\hline \multicolumn{12}{|l|}{ Fuerza } \\
\hline Hombres & 4.06 & 1.11 & \multirow{2}{*}{1.97} & \multirow{2}{*}{0.05} & 3.96 & 1.15 & 0.09 & 0.38 & -0.21 & \multirow{2}{*}{0.00} & \multirow{2}{*}{0.96} \\
\hline Mujeres & 3.59 & 1.05 & & & 3.57 & 1.00 & 0.02 & 0.32 & 0.28 & & \\
\hline \multicolumn{12}{|c|}{ Autoconcepto Físico General } \\
\hline Hombres & 4.10 & 1.07 & \multirow{2}{*}{2.44} & \multirow{2}{*}{$0.02^{*}$} & 4.15 & 0.98 & 0.05 & -0.26 & 0.35 & \multirow{2}{*}{1.76} & \multirow{2}{*}{0.19} \\
\hline Mujeres & 3.58 & 0.90 & & & 3.64 & 0.78 & 0.07 & -0.26 & 0.39 & & \\
\hline \multicolumn{12}{|l|}{ Autoconcepto General } \\
\hline Hombres & 4.48 & 0.70 & \multirow{2}{*}{2.04} & \multirow{2}{*}{$0.04^{*}$} & 4.56 & 0.65 & 0.11 & -0.17 & 0.39 & \multirow{2}{*}{3.14} & \multirow{2}{*}{0.08} \\
\hline Mujeres & 4.07 & 0.95 & & & 4.02 & 0.94 & 0.05 & 0.36 & -0.26 & & \\
\hline
\end{tabular}

\section{Discusión y conclusiones}

Los resultados obtenidos no permiten corroborar ninguna de las hipótesis planteadas en el estudio. Respecto a la primera, referente a la posibilidad de mejorar el autoconcepto físico en los jóvenes universitarios participantes en la intervención, se comprobó la ausencia de cambios estadísticamente significativos en las puntuaciones del grupo experimental tras la implementación del programa, tanto en el análisis intra-grupo como en la comparación con el grupo control (inter-grupo).

Son resultados que contrastan con los obtenidos en una implementación previa del programa (Axpe et al., 2013), sin embargo cabe recordar que en tal estudio los sujetos del grupo experimental simultanearon su tratamiento con una práctica física regular e intensa, inexistente en esta implementación. La ausencia de mejora resulta, por esta razón, relevante, ya que señalaría la importancia apuntada por otros autores (Marsh y Craven, 2006; Marsh et al., 2006) de complementar la intervención cognitiva con la conductual para lograr una mayor eficacia en la mejora del autoconcepto físico.
Por otro lado, intervenciones previas desde la prevención de los trastornos de la alimentación han mostrado la posibilidad de reducir la insatisfacción corporal de las personas participantes mediante intervenciones cognitivas (Anuel et al., 2012; Stice et al., 2011; Stice et al., 2012). A pesar de que la intervención del presente estudio fue cognitiva y multidimensional, es cierto también que en su implementación se adoptó una perspectiva educativodidáctica o informativa. Un tipo de aproximación que pudo no suponer la misma implicación personal y reestructuración cognitiva que los programas interactivos (Escoto et al., 2010) basados en la teoría de la disonancia cognitiva así como en la educación frente a los medios de comunicación (Góngora, 2010). Por lo que la ausencia de resultados de esta implementación sería relevante en el sentido de recordar lo necesario de cuidar el diseño y desarrollo de este tipo de intervenciones, favoreciendo actividades que contribuyan a la creación de disonancia cognitiva frente a perspectivas más informativas, a fin de lograr una fuerte implicación personal y que impacte a nivel cognitivo contribuyendo a cuestionar y modificar sus autopercepciones.

Es preciso, también, considerar la situación previa de 
los participantes, cuyas puntuaciones en autoconcepto físico antes del tratamiento resultaron elevadas, lo que por un lado dejaría poco margen de mejora y cambio estadísticamente significativo (O’Brien y LeBow, 2007) $\mathrm{y}$, por otro, podría haber contribuido a que, al no presentar problemas de insatisfacción relativos al yo físico, los sujetos no hubiesen percibido como relevante el contenido del programa (Nicolino et al., 2001) realizando por tanto sus tareas como algo rutinario (Escoto et al., 2010), lo que a su vez dificultaría la implicación personal y el cambio cognitivo.

En relación a la segunda hipótesis planteada, relativa a la mayor eficacia del programa "Mírate bien" en mujeres, por contar éstas con un menor autoconcepto físico, es preciso resaltar en primer lugar que el presente estudio corrobora, en línea con la investigación previa (Mayorga-Vega et al., 2012), la tendencia de los hombres a mostrar un mejor autoconcepto físico. En este trabajo son tres las escalas en las que dichas diferencias en función de sexo resultaron significativas: atractivo, autoconcepto físico general y autoconcepto general. Datos que parecen concordar con lo afirmado por otros autores en relación a la influencia mediática que provocaría, especialmente en las mujeres, una mayor insatisfacción con su imagen física al compararse con dichos ideales (Bermejo et al., 2011). Efectivamente, en nuestro estudio las mujeres obtienen, precisamente, peores puntuaciones en la dimensión de atractivo, así como en las escalas generales de autoconcepto físico y autoconcepto general, lo que apuntaría al gran peso que el aspecto físico puede llegar a tener sobre la autopercepción general (Infante et al., 2011; Lubans et al., 2010; Molero et al., 2013).

A pesar de las menores puntuaciones de partida de las mujeres, los datos del presente estudio no permiten confirmar que el programa educativo resulte más eficaz para mejorar su autoconcepto físico. En cualquier caso, el que no se constate diferencia alguna en la eficacia del programa en función de sexos es también un hecho relevante y positivo en el sentido de que, pese a no favorecer a las mujeres, éstas tampoco resultan perjudicadas $\mathrm{y}$ no valoran en peor medida su yo físico. Un problema observado en ciertas ocasiones cuando las jóvenes adquieren o se exponen a información relativa al físico y la apariencia (Bermejo et al., 2011). Esto hace que los materiales del "Mírate bien" puedan considerarse como una base útil y adecuada para futuras intervenciones siempre que éstas se diseñen desde una perspectiva cognitiva que favorezca una mayor implicación personal $\mathrm{y}$, por tanto, una mayor elaboración cognitiva de los participantes.

Para finalizar, es preciso tener en cuenta las limita- ciones del presente trabajo, entre las que se cuenta el tamaño y distribución de la muestra, la falta de medidas de seguimiento así como la ausencia de registros relativos a variables que podrían arrojar una mayor información acerca de la eficacia del programa en la modificación de hábitos de vida saludables o respecto a la satisfacción vital de los participantes.

Todas estas cuestiones deberán ser tenidas en cuenta en investigaciones futuras, a fin de lograr determinar las características más adecuadas tanto en el diseño como en la implementación de intervenciones para la mejora de una variable de tal relevancia y repercusión en el bienestar y ajuste personal y social de los sujetos como lo es el autoconcepto físico. 


\section{Referencias}

Annesi, J. J, Unruh, J. L., Marti, N. C., Gorjala, S. y Tennant, G. (2011). Effects of the Coach approach intervention on adherence to exercise in obese women. Assessing mediation of Social Cognitive theory factors. Research Quarterly for Exercise and Sport, 82(1), 99-108. doi: $10.1080 / 02701367.2011 .10599726$

Anuel, A., Bracho, A., Brito, N., Rondón, J. E. y Sulbarán, D. (2012). Autoaceptación y mecanismos cognitivos sobre la imagen corporal [Cognitive mechanisms and self-acceptance of body image]. Psicothema, 24(3), 390-395.

Axpe, I., Goñi, A. e Infante, I. (2013). Efficacy of a cognitive program for improving the physical selfconcept of university students. Perceptual and Motor Skills, 117(3), 1-13. doi: 10.2466/06.27.PMS.117x32z9

Babic, M. J., Morgan, P. J., Plotnikoff, R. C., Lonsdale, C., White, R. L. y Lubans D. R. (2014). Physical activity and physical self-concept in youth: systematic review and meta-analysis. Sports Medicine, 44(11), 1589-1601. doi: 10.1007/s40279-014-0229-z

Bermejo, B. G., Saúl, L. A. y Jenaro, C. (2011). La anorexia y bulimia en la red: Ana y Mia dos malas compañías para las jóvenes de hoy [The anorexia and bulimia on the web: Ana and Mia, two "bad company" for youth today]. Acción Psicológica, 8(1), 71-84.

Borrego F. J., López-Sánchez, G. y Díaz-Suárez, A. (2012). Influencia de la condición física en el autoconcepto de un conjunto de adolescentes del municipio de Alcantarilla [Physical condition influence in self-concept of a teens group of Alcantarilla town]. Cuadernos de Psicología del Deporte, 12(2), 57-62.

Ceschini, F. L., Andrade, D. R., Oliveira, L. C., Araujo, J. F. y Matsudo, V. K. R. (2009). Prevalence of physical inactivity and associated factors among high school students from state's public schools. Jornal de Pediatria, 85(4), 301-306. doi: $10.1590 / \mathrm{S} 0021-75572009000400006$

Cava, M. J., Musitu, G. y Vera, A. (2000). Efectos directos e indirectos de la autoestima en el ánimo depresivo [Direct and indirect effects of self-esteem on depressive mood]. Revista Mexicana de Psicología, 17(2), 151-161.
Celio, A., Goldschmit, A., Huang, C., Winzelberg, A. J., Taylor, C. B. y Story, M. (2008). Reduction of overweight and eating disorder symptoms via the Internet in adolescents: A randomized controlled trial. Journal of Adolescent Health, 43(2), 172-179. doi: 10.1016/j.jadohealth.2008.01.011

Chanal, J., Marsh, H., Sarrazin, P. y Bois, J. (2005). Bigfish-little-pond effects on gymnastics self-concept: Social comparison processes in a physical setting. Journal of Sport and Exercise Psychology, 27(1), 5370.

Cohen, J. (1988). Statistical power analysis for the behavioral sciences. Hillsdade, N.J: Lawrence Erlbaum Associates.

Contreras, O. R., Fernández, J. G., García, L. M., Palou, P. y Ponseti, J. (2010). El autoconcepto físico y su relación con la práctica deportiva en estudiantes adolescentes [Relationship in adolescents between physical self-concept and participating in sport]. Revista de Psicología del Deporte, 19(1), 23-39.

Dimitrov, D. M. y Rumrill, P. D. (2003). Pretest-posttest designs and measurement of change. Work, 20, 159165.

Escoto, M. C., Camacho, E. J., Rodríguez, G. y Mejía, J. (2010). Programa para modificar Alteraciones de la Imagen Corporal en estudiantes de Bachillerato [Program to modify Body Image disturbances in HighSchool students]. Revista Mexicana de Trastornos Alimentarios/Mexican Journal of Eating Disorders, 1, 112-118.

Esnaola, I. y Goñi, A. (2006). Autokontzeptu fisikoa neurtzeko galdesorta berri baten propietate psikometrikoak: Autokontzeptu Fisikoaren Itaunketa (AFI) [Psychometric properties of a new questionnaire to assess physical self concept: Physical Self Concept Questionnaire (AFI)]. Uztaro, 56, 109-122

Esnaola, I. (2007). Alimentación, autoconcepto e imagen corporal [Diet, self-concept and body image]. Revista de Psicología Social, 22(2), 149-163.

Esnaola, I., Infante, G. y Zulaika, L. (2011). The Multidimensional Structure of Physical Self-Concept. The Spanish Journal of Psychology, 14(1), 304-312.

Esnaola, I., Rodríguez, A. y Goñi, A. (2010). Body dissatisfaction and perceived sociocultural pressures: 
gender and age differences. Salud Mental, 33, 21-29.

Esnaola, I. y Zulaika, L. M. (2009). Physical activity and physical self-concept in a sample of middle-age basque adults. Perceptual and Motor Skills, 108, 479-490. doi: 10.2466/pms.108.2.479-490

Fernández, J. G., Contreras, O. R., García, L. M. y González, S. (2010). Autoconcepto físico según la actividad físicodeportiva realizada y la motivación hacia ésta [Physical self-concept depending on physical activity practiced and motivation to it]. Revista Latinoamericana de Psicología, 42(2), 251-263.

Fernández-Bustos, J. G., González-Martí, I., Contreras, O. y Cuevas, R. (2015). Relación entre imagen corporal y autoconcepto físico en mujeres adolescentes [Relation between body image and physical self concept of adolescent women]. Revista Latinoamericana de Psicología, 47(1), 25-33.

Fox, K. R. y Corbin, C. B. (1989). The Physical SelfPerception Profile: development and preliminary validation. Journal of Sports and Exercise Psychology, 11(4), 408-430.

Franko, D. L. y Striegel-Moore, R. H. (2002). The role of body dissatisfaction as a risk factor for depression in adolescent girls: Are the differences Black and White? Journal of Psychosomatic Research, 53, 975983.

García-Alandete, J. y Bernabé-Valero, G. (2013). Religious Orientation and Psychological Well-Being among Spanish Undergraduates [Orientaciones religiosas y bienestar psicológico de los estudiantes universitarios españoles]. Acción Psicológica, 10(1), 135-148. http://dx.doi.org/10.5944/ap.10.1.7040

Gasco, M., Briñol, P. y Horcajo, J. (2010). Cambio de actitudes hacia la imagen corporal: el efecto de la elaboración sobre la fuerza de las actitudes [Attitude change toward body image]. Psicothema, 22(1), 7176.

Góngora, V. (2010). Hacia una integración de los paradigmas positivos y de enfermedad en la prevención de los trastornos de la conducta alimentaria en adolescentes [Towards an integration of positive- and illnes- paradigms in the prevention of Eating Behavior Disorders in adolescents]. Psicodebate, 10, 279296.
González-Brignardello, M. P. y Sánchez-Elvira-Paniagua, A. (2013). ¿Puede amortiguar el engagement los efectos nocivos de la procrastinación académica? [Can Engagement buffer the harmful effects of Academic Procrastination?]. Acción Psicológica, 10(1), 117-134. http://dx.doi.org/10.5944/ap.10.1.7039

Goñi, A. y Rodríguez, A. (2007). Variables associated with the risk of eating disorders in adolescence. Salud Mental, 30(4), 16-23.

Goñi, A., Ruiz de Azúa, S., y Rodríguez, A. (2006). Cuestionario del Autoconcepto Físico. Manual [Physical Self Concept Questionnaire. Manual]. Madrid, España: EOS.

Goñi, E. y Infante, G. (2010). Actividad físico-deportiva, autoconcepto físico y satisfacción con la vida [Physical-sporting activity, physical self-concept and satisfaction with life]. European Journal of Education and Psychology, 3(2), 199-208.

Guedes, D. P., Souza, V. M., Ferreirinha, J. E. y Silva, J. R. M. (2012). Physical activity and determinants of sedentary behavior in Brazilian adolescents from an underdeveloped region. Perceptual and Motor Skills, 114(2), 542-552. doi: 10.2466/06.13.17.PMS.114.2.542-552

Haugen, T., Ommundsen, Y. y Seiler, S. (2013). The relationships between physical activity and physical self-esteem in adolescents: the role of physical fitness indices. Perspectives Pediatric Exercise Science, 25, 138-153.

Infante, G., Axpe, I., Revuelta, L. y Ros, I. (2012). Autopercepción física y modalidades de actividades físicas en la edad adulta [Physical self-perceptions and physical activity patterns in adulthood]. APUNTS. Educación Física y Deportes, 110, 19-25.

Infante, G., Goñi, A. y Villaroel, J. D. (2011). Actividad física y autoconcepto, físico y general, a lo largo de la edad adulta [Physical activity and physical and general self-concept during adulthood]. Revista de Psicología del Deporte, 20(2), 429-444.

Jenaro, C., Flores, N., Bermejo, B. G. y Cruz, M. (2011). Body Image Questionnaire for early detection of Eating Disorders [Cuestionario de Imagen Corporal para la detección temprana de Trastornos de la Conducta Alimentaria]. Acción Psicológica, 8(1), 7-20.

Lindwall, M., Asçi, H., Crocker, P. y Teixeira, P. J. (2014). The physical self in motion: within-person 
change and associations of change in self-esteem, physical self-concept and physical activity in adolescent girls. Journal of Sport y Exercise Psychology, 36(6), 551-563. doi: 10.1123/jsep.2013-0258

Lubans, D. R., Aguiar, E. J. y Callister, R. (2010). The effects of free weights and elastic tubing resistance training on physical self-perception in adolescents. Psychology of Sport and Exercise, 11, 497-504. doi:10.1016/j.psychsport.2010.06.009

Marsh, H. W. y Craven, R. G. (2006). Reciprocal effects of self-concept and performance from a multidimensional perspective: Beyond seductive pleasure and unidimensional perspectives. Perspectives on Psychological Science, 1(2), 133-163. doi: $10.1037 / \mathrm{h} 0050099$

Marsh, H. W., Papaioannou, A. y Theodorakis, Y. (2006). Causal ordering of physical self-concept and exercise behavior: Reciprocal effects model and the influence of physical education teachers. Health Psychology, 25, 316-328. doi: 10.1037/02786133.25.3.316

Mayorga-Vega, D., Viciana, J. y Cocca, A. (2012). Relationships between physical self-concept and health-related physical fitness in Spanish schoolchildren. Procedia - Social and Behavioral Sciences, 69, 659-668. doi:10.1016/j.sbspro.2012.11.458

Molero, D., Zagalaz-Sánchez, M. L. y Cachón-Zagalaz, J. (2013). Estudio comparativo del autoconcepto físico a lo largo del ciclo vital [Comparative study based on the physical self-concept in teenagers regarding gender and physical activity]. Revista de Psicología del Deporte, 22(1), 135-142.

Nicolino, J. C., Martz, D. M. y Curtin, L. (2001). Evaluation of a cognitive-behavioral therapy intervention to improve body image and decrease dieting in college women. Eating Behaviors, 2, 353-362. doi:10.1016/S1471-0153(01)00043-5

O’Brien, K. M. y LeBow, M. D. (2007). Reducing maladaptive weight management practices: developing a psycho-educational intervention program. Eating Behaviors, $8, \quad 195-210$. doi:10.1016/j.eatbeh.2006.06.001

Parker, P., Martin, A. y Marsh, H. (2008). Factors predicting life satisfaction: A process model of personality, multidimensional self-concept, and life satisfaction. Australian Journal of Guidance y Counsel- ing, 18(1), 15-29. doi: 10.1375/ajgc.18.1.15

Rahmani-Nia, F., Damitchi, A., Azizi, M. y Hoseini, R. (2011). Associations between self-perceived and measured physical fitness of male college students. World Applied Sciences Journal, 14(9), 1331-1338.

Revuelta, L., Esnaola, I. y Goñi, A. (2013). El autoconcepto físico como determinante de la actividad físicodeportiva durante la adolescencia [Physical self concept as a determinant of physical and sport activity during adolescence]. Psicología Conductual, 21(3), 581-601.

Rodríguez-Fernández, A., González-Fernández, O. y Goñi, A. (2013). Sources of perceived sociocultural pressure on physical self-concept. Psicothema, 25, 192-198. doi:10.7334/psicothema2012.229

Sánchez-Meca, J. (2008). Meta-análisis de la investigación [Meta-analysis in research]. En M. A. Verdugo et al. (Coords.), Metodología en la investigación sobre discapacidad [Metodology in disability research] (pp. 121-139). Salamanca, España: Publicaciones del INICO.

Sánchez-Quintero, S. y De la Vega, I. (2013). Introducción al tratamiento basado en la mentalización para el trastorno límite de la personalidad [Introduction to the mentalization-based treatment for borderline personality disorder]. Acción Psicológica, 10(1), 21-32. http://dx.doi.org/10.5944/ap.10.1.7030

Scarpa, S. (2011). Physical self-concept and self-esteem in adolescents and young adults with and without physical disability: the role of sports participation. European Journal of Adapted Physical Activity, 4(1), 3853.

Schmidt, M., Valkanover, S., Roebers, C. y Conzelmann, A. (2013). Promoting a functional physical selfconcept in physical education: Evaluation of a 10week intervention. European Physical Education Review, 19(2), 232-255. doi: $10.1177 / 1356336 \times 13486057$

Soriano, J. A., Navas, L. y Holgado, F. P. (2011). El autoconcepto físico y su relación con el género y la edad en estudiantes de educación física [Physical selfawareness and its relationship with gender and age in physical education students]. APUNTS. Educación Fisica y Deportes, 106, 36-41.

Shavelson, R. J., Hubner, J. J. y Stanton, J. C. (1976). Self concept: validation of construct interpretations. 
Review of Educational Research, 46, 407-441. doi: $10.3102 / 00346543046003407$

Soriano, J. A., Sampascual, G. y Navas, L. (2010). El autoconcepto físico en la clase de Educación Física: un análisis centrado en el sexo [Self-concept in physical education classroom: an analysis focused on sex]. International Journal of Developmental and Educational Psychology. INFAD. Revista de Psicología, 3(1), 419-425.

Stice, E., Marti, N., Rohde, P. y Shaw, H. (2011). Testing mediators hypothesized to account for the effects of a dissonance eating disorder prevention program over longer-term follow-up. Journal of Consulting and Clinical Psychology, 79, 398-405. doi: 10.1037/a0023321

Stice, E., Rohde, P., Durant, S. y Shaw, H. (2012). A preliminary trial of a prototype Internet dissonancebased eating disorder prevention program for young. Journal of Consulting and Clinical Psychology, 80(5), 907-916. doi: 10.1037/a0028016

Stice, E. y Shaw, H. (2004). Eating disorder prevention programs: A meta-analytic review. Psychological Bulletin, 130, 206-227. doi: 10.1037/00332909.130.2.206

Vancampfort, D., Probst, M., Sweers, K., Maurissen, K., Knapen, J. y De Hert, M. (2011). Relationships between obesity, functional exercise capacity, physical activity participation and physical self-perception in people with schizophrenia. Acta Psychiatrica Scandinavica, 123, 423-430. doi: 10.1111/j.16000447.2010.01666.x

Vedul-Kjelsås, V., Sigmundsson, H., Stensdotter, A. K. y Haga, M. (2012). The relationship between motor competence, physical fitness and self-perception in children. Child Care, Health and Development, $38(3)$, 394-402. doi: 10.1111/j.13652214.2011.01275.x

Velez, A., Golem, D. L. y Arent, S. M. (2010). The impact of a 12-week resistance training program on strength, body composition, and self-concept of Hispanic adolescents. Journal of Strength and Conditioning Research, 24(4), 1065-1073. doi: 10.1519/JSC.0b013e3181cc230a

Videra-García, A. y Reigal-Garrido, R. (2013). Autoconcepto físico, percepción de salud y satisfacción vital en una muestra de adolescentes [Physical self- concept, perceptions of health and life satisfaction in a sample of adolescents]. Anales de Psicología, 29(1), 141-147. doi: 10.6018/analesps.29.1.132401 